\title{
Feature Extraction for Robust Crack Monitoring Using Passive Wireless RFID Antenna Sensors
}

\author{
Jun Zhang, Member, IEEE, Ali Imam Sunny, Gary Zhang, Senior Member, IEEE, and Guiyun Tian, \\ Senior Member, IEEE
}

\begin{abstract}
Radio frequency identification (RFID) tag antenna based passive wireless sensors are receiving increasing attentions for structural health monitoring (SHM) in large-scale infrastructure. For permanently-installed monitoring, robustness to measurement variation including environmental conditions is a practical issue. This paper retrospects the communication principle of magnetic resonant coupling (MRC) for low frequency (LF) passive wireless RFID antenna sensors. The influence of communication is uncovered and essentially separated from sensing through two steps: sweep (source) frequency to capture the system behavior, i. e., resonance frequency range; multiple feature extraction, fusion, and selection in conjunction with normalization for the selected time-domain feature of peak to peak (P2P). By this way, the robustness of the low-cost RFID sensing system is enhanced. The proposed method is validated by the case study in open crack detection and characterization under varied measurement conditions.
\end{abstract}

Index Terms-Magnetic resonance coupling (MRC), crack characterization, sweep frequency, radio frequency identification (RFID), structural health monitoring (SHM), time-frequency feature extraction.

\section{INTRODUCTION}

$\mathrm{N}$ ON-destructive testing and evaluation (NDT\&E) is widely used in industry for reliability and safety of structures. Cracks are common mechanical failures [1]. While small cracks may lead to inadequate serviceability, large cracks can result in structural failures [2]. Many structural health monitoring (SHM) techniques, such as ultrasonic wave and fiber Bragg strain sensors, are adopted for this purpose. An exponential increase of infrastructure demands a reduction in the cost and size of conventional sensing techniques for continuously monitoring in their life cycle. Reliable and passive sensors with small size and power harvesting capability are well on its way [3], which should be permanently installed and detect, locate, and quantify defects on-demand. This is a

Manuscript received on March 20, 2018; revised on June 3, 2018. This work was supported in part by the National Natural Science Foundation of China (NSFC) under grant no. 61701120 and in part by the Novel Sensing Networks for Intelligent Monitoring (NEWTON) project from EPSRC under grant no. $\mathrm{EP} / \mathrm{J} 012343 / 1$.

Jun Zhang and Gary Zhang are with the School of Information Engineering, Guangdong University of Technology, Guangzhou 510006, China. (e-mail: junzhang(g,gdut.edu.cn)

Ali Imam Sunny and Guiyun Tian are with the School of Electrical and Electronic Engineering, Newcastle University, Newcastle upon Tyne, NE1 7RU, United Kingdom. (e-mail: g.y.tian@ncl.ac.uk) major motivation for bridging NDT\&E and SHM through the extension of NDT systems for in-situ online monitoring [4].

The pulsed eddy current (PEC) technique, that is composed of excitation and pickup coils [5], is widely used for NDT\&E purpose. The step response received in the pickup coil has rich information than multi-frequency eddy current [6]. Similar sensing mechanism is found for antenna sensors. A sensitivity of $22.1 \mathrm{MHz} / \mathrm{mm}$ was achieved using a patch antenna for crack monitoring in the microwave region [7]; however, an accurate vector network analyzer (VNA) is required to obtain the reflection coefficient. The cost benefit of passive wireless antenna sensors enabling with radio frequency identification (RFID) technology has gained much attention in both academy and industry for potential monitoring applications in both structure health and environmental conditions [8]. Armed with unique identification (UID), this type of sensor is a good candidate for densely distributed sensing application [9].

In the ultra-high frequency (UHF) band, a patch antenna was used for strain and emulated crack monitoring, where the turn-on power was used as a sensing parameter in a distance of $2.1 \mathrm{~m}$ between reader and tag antennas [10]. A sensitivity of $-1.19 \times 10^{4} \mathrm{ppm} / \mathrm{mm}$ can be achieved using the feature of resonant frequency shift. However, the installation of the large size antenna may affect the mechanical behavior of structure. In conjunction with backscattered phase measurement, two coupled RFID antenna sensors were developed for crack width characterization [11]. A sub-mm crack enlargement can be detected using a commercial UHF reader in a distance of $1.5 \mathrm{~m}$. Nevertheless, the distance and orientation between reader and tag are not allowed to vary during the whole monitoring cycle because the phase measurement is dependent on the wireless channel. Recently, an ultra-wide band (UWB) chip-less antenna was developed for crack width monitoring, where backscattered power was measured with a distance up to $30 \mathrm{~cm}$ in an anechoic chamber [12]. Depending on the position of the crack orientation relative to the antenna, the sensor has a positive or negative coefficient in the feature of resonant frequency shift. Regardless the resolution is improved with scale down of wavelength as frequency increases, large crack depth may not be identified using a proximity coupling sensing mechanism because of the decrease in penetration depth [13].

An open-ended waveguide (OEWG) was used to interrogate a wireless passive sensor for monitoring temperature up to $1000^{\circ} \mathrm{C}$ and it was experimentally validated to capture the resonant behavior of antenna sensor with a time-domain (TD) gating method in a distance of $30 \mathrm{~mm}$ [14]. Above all, the 
design of low-cost interrogators are receiving increasing attentions to make sensing system practical. Rather than using an expensive but cumbersome VNA, a low-cost UWB radar was developed to interrogate with time-coded chipless tag sensors, where temperature was wirelessly obtained from the tag mode amplitude of the backscattered signals in a distance up to $1 \mathrm{~m}$ [15]. Based on a time-domain radar-based interrogator, a microwave dielectric resonator was designed to monitor the temperature up to $700^{\circ} \mathrm{C}$ in a distance of $1.2 \mathrm{~m} \mathrm{[16]}$. An interrogation system that was able to acquire the sensing signal in the far field was developed and the temperature up to $280^{\circ} \mathrm{C}$ was wirelessly acquired in a distance of $60 \mathrm{~mm}$ [17]. Recently, a frequency modulated continuous wave (FMCW) radar was developed to interrogate antenna sensors [18], which is able to extract the resonant frequency in real time.

Comparing with its UHF and UWB counterparts, the low frequency (LF) RFID uses non-radiative electromagnetic for communication, which is less influenced by wireless channel but at a sacrifice of the communication distance. The LF RFID tag coil has a better ability to read near metal or wet surfaces [19]. Also, the tag coil that always consists of $\mathrm{N}$ number of loops can be used for monitoring crack in any orientation. However, the response from the load modulation of the tag in the reader is dominated by the circuit and magnetic resonant coupling (MRC) between the reader and tag rather than eddy current induced by the tag only. The feature in the steady state is, therefore, influenced by the system behavior of MRC based wireless power transfer (WPT), such as quality factor and communication distance (or mutual coupling) between the reader and tag coils. In ubiquitous monitoring, the mutual coupling varies due to system characters. Hence, it is a practical issue for the LF RFID sensing system to adopt the variation.

It is known that the influence from the lift-off distance can be partially removed in the PEC technique through normalization of the step response [20-22]. To improve the robustness of RFID sensing system is critical in defect monitoring, which can improve the system reliability and therefore reduce the false alarm or false dismissal. Monitoring resonant frequency of antenna sensor is a straightforward but effective way for defect characterization as above mentioned. In addition, a principal component analysis (PCA) method was proposed to mitigate the distance dependence of a high frequency (HF) RFID sensing system for corrosion monitoring [23]. However, the method requires a cumbersome VNA for accurate impedance measurement in the frequency domain.

This paper aims to improve the robustness of low-cost passive wireless RFID antenna sensors for potential SHM through sweeping frequency to capture the system behavior and mitigate distance influence. This paper is organized as follows. Sensing mechanism and communication principle based on MRC are revisited in Section II, where the influence of mutual coupling is uncovered for the LF RFID sensing system. A feature extraction, fusion, and selection procedure is introduced to separate the communication from sensing in this section as well. Section III presents a case study to validate the proposed method. Finally, Section IV concludes the research findings.

\section{CommunicAtion AND SENSING PRINCIPLES}

The possible types of interference for measurement include system configurations such as circuit bandwidth and communication distance, sample influences such as surface roughness or sample geometry, thickness, permittivity, and permeability, and environmental conditions such as operating temperature [24]. In a real environment, the defect and interference will behave as a combination of multiple types. The inversion for the RFID sensing system, therefore, is an ill-posed problem. This section first retrospects the sensing and then communication principles of LF RFID sensing system based on MRC. After that, a time-frequency feature extraction, fusion, and selection procedure is introduced to separate the influence of communication distance from sensing.

\section{A. Sensing principle}

The theory behind using a tag coil to detect metal with defect (i.e., target) can be equivalent to the complex image method [25]: the magnetic field created by the sensor $\left(\mathrm{H}_{1}\right)$ induces eddy currents in the target, which in turn generates a secondary magnetic field $\left(\mathrm{H}_{2}\right)$ opposing the initial one. As a result, a magnetic coupling (represented by the mutual inductance $\mathrm{M}_{12}$ ) appears between the tag coil and target.

The principle of RFID antenna sensor based on the tag coil can be described in [26]. For a straight wire of length $l$, height $h$ above a perfectly conducting screen, the mutual partial inductance is [27]

$$
M_{p 12}^{\text {wire/screen }} \approx \frac{\mu_{0}}{2 \pi} l\left(\ln \left[\frac{l}{h}\right]-1\right),
$$

where the approximation is good for $l>>h$. Applying complex image method for a conductive slab, $h$ can be replaced by $h+D$, where the second term is determined by the penetration depth, i.e., $D=\sqrt{2 / \omega \mu \sigma}$. Therefore, the partial impedance of the wire can be represented as

$$
Z_{p}^{\text {wire } / \text { slab }}=R_{p 1}+j \omega\left(L_{p 11}^{\text {wire }}-M_{p 12}^{\text {wire/slab }}\right) .
$$

Where $L_{p 11}^{\text {wire }}$ is the self-inductance of the wire. The sensing information is contained in the mutual inductance of $M_{p 12}^{\text {wire } / s l a b} . R_{p 1}$ is the loss of the wire, and it also includes the eddy current induced loss.

When the target moves away, the mutual inductance decays, leading an increase of self-inductance of the tag coil [28]. At the same time, the parasitic series resistance of the tag coil slightly decays. This can be explained by Lenz's law: moving the target away implies a smaller secondary magnetic field and hence a smaller amount of eddy current or less dissipated energy (i.e., equivalent coil resistance lowers) [29]. Typically, the change in $R_{p 1}$ is negligible when compared to the inductive variations as the target moves away.

\section{B. Reader and interrogation method}

Communication and sensing are a contradiction in the LF RFID sensing systems. Both of them are in the near-field region while the former is based on MRC and the latter is relying on 


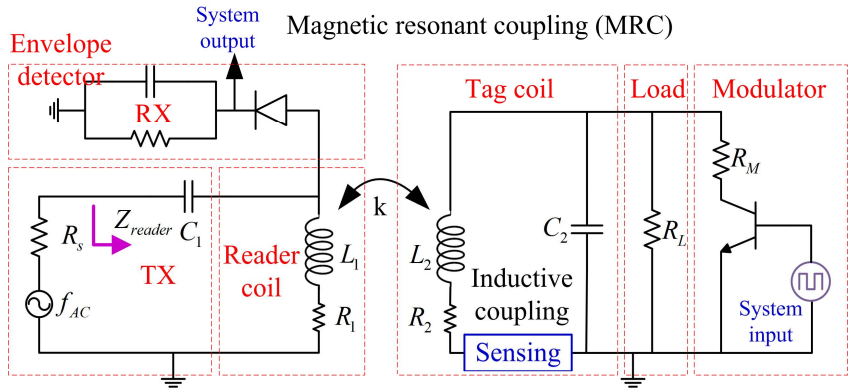

(a)

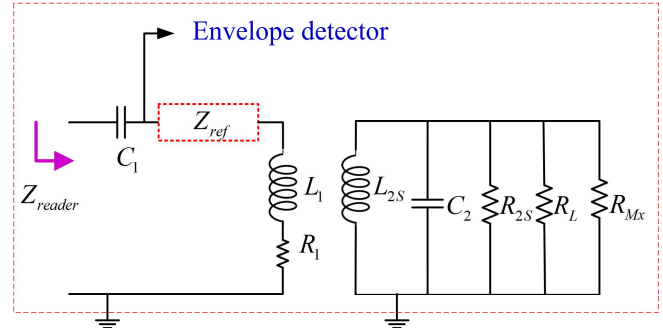

(b)

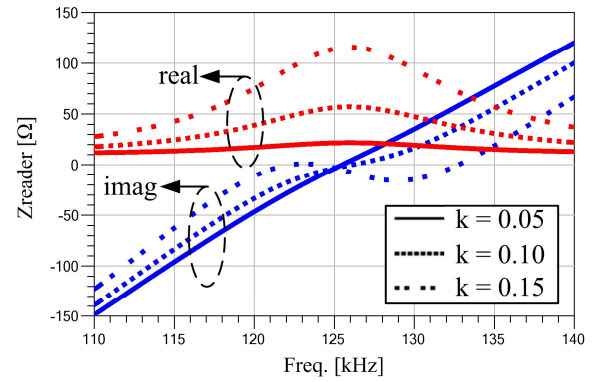

(c)

Fig. 1. WPT with an SP topology for LF RFID sensing system (a) system configuration, (b) equivalent circuit, and (c) reader impedance.

the magnetic inductive coupling. To improve the efficiency of WPT, the quality factors of the reader and tag coils are the higher, the better. However, from the communication point of view, the high quality factor leads a very narrow bandwidth and therefore limits the achievable data rate. The similar trade-off can be found in UHF RFID sensing system [30] and also in simultaneous wireless information and power transfer (WIPT), which is originally developed for 5G cellular network [31].

The topology for WPT in the LF RFID sensing system is equivalent to a series-parallel (SP) structure. The research in WPT can be in general divided into five categories [32], among which the allowance of no magnetic coupling $(k=0)$ is desired for sensing purpose. The power transfer efficiency (from source to load) is one of the more widely investigated performance characterizations of non-radiative WPT [33]. When the link is loosely coupled $(k<<1)$ [34], the power transfer efficiency is low. For a long communication distance, due to the free of frequency splitter in a strong coupling [35], a critical coupling is an objective to maximize the power transfer efficiency [36].

In this paper, a sweep-frequency reader has been used to get the system resonance behavior. The interrogation method is described in Fig. 1(a), where $L_{2}$ and $R_{2}$ are the self- inductance and resistance of the tag coil. The operating frequency of the RFID sensing system can be changed by varying the frequency of the AC source $\left(f_{A C}\right)$. The equivalent circuit of the system is shown in Fig. 1(b). In parallel connected with a capacitor and two resistors, the inductor of the tag coil can form an RLC parallel resonance, whose quality factor can be written as

$$
Q_{\text {tag }}=\frac{R_{\text {tag }}}{\omega_{0} L_{2 S}} .
$$

The resistance $R_{\text {tag }}$ is determined by the three parallel resistors: power demand of the tag, i.e., $R_{L}$, the switched one connected into load modulation, i.e., $R_{M x}$, and the loss of the coil, i.e., $R_{2 S}=\omega_{0}^{2} L_{\text {tag }}^{2} / R_{2}$, which is originally connected in series with the tag coil. The subscript $x$ represents the on and off states for the load modulation [37]. The sensing information is reflected in the tag coil's inductance $\left(L_{2 S}\right)$ and lossy resistance $\left(R_{2 S}\right)$, as indicated in (2). The system resonance behavior is depicted in Fig. 1(c). It can be observed that the resonant frequency has a negligible dependence on the mutual coupling (or communication distance) between the reader and tag in under coupling condition.

From reader point view, the effect of magnetic coupling with a tag coil can be represented as an equivalent impedance value called reflected impedance, i.e., $Z_{\text {ref }}=(\omega M)^{2} / Z_{\text {tag }}$ [38]. The input impedance of tag $\left(Z_{\text {tag }}\right)$ is

$$
\frac{1}{Z_{\text {tag }}}=\frac{1}{j \omega L_{2 S}}+j \omega C_{2}+\left(\frac{1}{R_{L}}+\frac{1}{R_{M}}+\frac{1}{R_{2 S}}\right) .
$$

Here, the mutual inductance between the reader and tag coils is $M=k \sqrt{L_{1} L_{2 S}}$. The influence of the communication distance can be modeled as coupling coefficient of $k$ in the frequency domain [39]. The input impedance in the reader $\left(Z_{\text {reader }}=\right.$ $Z_{1}+Z_{\text {ref }}$ ) can, therefore, be detailed as

$$
\begin{aligned}
Z_{\text {reader }} & =\left(\frac{1}{j \omega C_{1}}+j \omega L_{1}+R_{1}\right)+\omega^{2} k^{2} L_{1} L_{2 S} \times \\
& \times\left[\frac{1}{j \omega L_{2 S}}+j \omega C_{2}+\left(\frac{1}{R_{L}}+\frac{1}{R_{M x}}+\frac{1}{R_{2 S}}\right)\right]
\end{aligned}
$$

Unlike that of its parallel-resonant counterpart, we can see from (5) that the output voltage of the series-resonant reader is sensitive to coupling variation [38]. The tag's loaded-Q and the reader's inductance should be large in order to obtain high reflected resistance under a given coupling coefficient. However, the parameters of the series resonance in the reader side influence the extraction of tag's sensing information.

When both reader and tag coils resonate, the voltage input to the envelope detector can be written as

$$
V_{\text {dem }}=V_{0}\left(1+j Q_{S L}-R_{S} / R_{t o t}\right),
$$

where $V_{0}$ is the amplitude of the AC source, and $Q_{S L}$ is the quality factor in the reader, which is determined by the loss in the reader coil as well as the resistance in the tag coil as

$$
Q_{S L}=\omega_{0} L_{1} / R_{t o t} \text {. }
$$

Here,

$$
R_{t o t}=R_{S}+R_{1}+\omega^{2} k^{2} L_{1} L_{2 S}\left(\frac{1}{R_{L}}+\frac{1}{R_{M x}}+\frac{1}{R_{2 S}}\right) .
$$

In this case, the power transfer efficiency from the source to the tag can be written as 


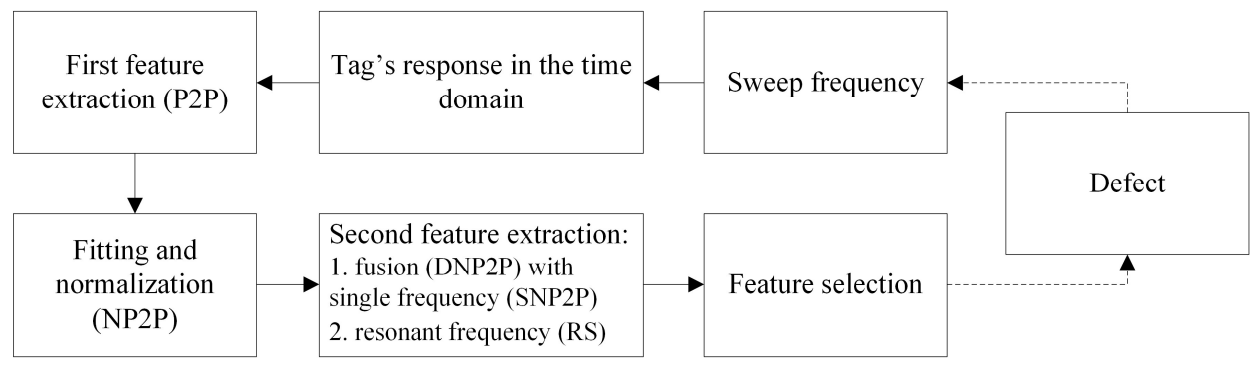

Fig. 2. Procedure of time-frequency feature extraction, fusion, and selection procedure.

$$
\begin{aligned}
\eta_{\text {eff }}= & \frac{R_{\text {ref }}}{R_{S}+R_{1}+R_{\text {ref }}} \\
= & \frac{\omega^{2} k^{2} L_{1} L_{2 S}\left(\frac{1}{R_{L}}+\frac{1}{R_{M x}}+\frac{1}{R_{2 S}}\right)}{R_{S}+R_{1}+\omega^{2} k^{2} L_{1} L_{2 S}\left(\frac{1}{R_{L}}+\frac{1}{R_{M x}}+\frac{1}{R_{2 S}}\right)} .
\end{aligned}
$$

When $R_{\text {ref }}<R_{S}=50 \Omega$, the reflected resistance increases with the coupling coefficient, leading an increase in the power transfer efficiency.

\section{Feature extraction, fusion, and selection}

The basic principle of LF RFID sensing system is to detect changes of the capacitance, resistance, or inductance of the tag coil in response to the parameter of interest (e.g., crack). This detection procedure can be realized by monitoring the shift in the resonant frequency, input impedance, or quality factor of system behavior through tag's response in the reader [40]. However, the shift may be caused by variations of system configurations, environmental condition, or defect.

In general, the features of $\Delta Z_{M x}, \Delta Q, \Delta f$ can be extracted from the tag's response in the reader to characterize the physical parameter of interest. Here, $\Delta$ represents the variation in system characters, including the communication distance or crack defect. The variations of impedance and quality factor are dependent on $\Delta k$, which means the communication distance may influence their sensitivities in the signal acquisition. However, $\Delta f$ can be used to mitigate the influence of the distance, which is relying on $\Delta L, \Delta C, \Delta R$ or equally the perturbation of defect in the inductance, capacitance, or resistance of the tag coil.

The procedure of the time-frequency feature extraction, selection, and fusion proposed in this paper is displayed in Fig. 2 . First, the frequency of the reader is swept to trace the system resonance behavior. The tag's response in the time domain is wirelessly captured in the reader. The feature of $\Delta Z_{M}=\Delta Z_{M 1}$ $\Delta Z_{M 0}$ can be indirectly measured through peak to peak (P2P) of the envelope in tag's response when the amplitude of the source voltage is fixed. The extraction for the time-domain feature of $\mathrm{P} 2 \mathrm{P}$ can be found in [41]. To suppress the influence of the coupling coefficient as well as the noise, $\mathrm{P} 2 \mathrm{P}$ is firstly fitted using the polynomial curve fitting technique and then normalized to its maxima (resonant frequency point) over the swept frequency range. The normalization is followed by a fusion procedure, where the differential is taken on both sides of the resonant frequency point. A feature of resonant frequency will also be extracted from the time-frequency feature representation using the normalized P2P (NP2P). The performance of the extracted features will be analyzed and selected in the following section.

\section{CRACK DETECTION AND CHARACTERIZATION}

\section{A. Experimental setup}

An LF RFID reader with adjustable source frequency has been utilized to perform the experimental work. Figs. 3(a-b) show the experimental setup to capture the system resonance behavior. A signal generator was used as a tunable source to prove the concept. In fact, the signal generator can be replaced by a voltage controlled oscillator (VCO) with a $\mu$-controller as an integrated device [42]. The reader coil used was $90 \mathrm{~mm}$ in diameter with parameters of $\mathrm{Rr}=10.45 \Omega$ and $\mathrm{Ls}=732 \mu \mathrm{H}$. The tag used was 231 Volcano LF RFID tag, which resonates at $125.0 \mathrm{kHz}$ [43]. The tag was programmed with an ID consisting of ' 1 ' with a $50 \%$ duty cycle at a data rate of one $32^{\text {nd }}$ of source frequency. The signal from the reader coil was demodulated by the internal circuitry to obtain the envelope of tag's response. The demodulated signal was then sampled using 14-bit Adlink 2010 DAQ. The data acquisition was controlled in the LABVIEW platform. For each test, the output of the reader was sampled at $1 \mathrm{MHz}$ for a period of $0.01 \mathrm{~s}$, i.e., 10,000 samples per test. To improve the signal to noise ratio, the tag's response in the time domain was divided into bits and then averaged.

The material of the defect sample is aluminum with a conductivity of $25.0 \mathrm{MS} / \mathrm{m}$. The sample has five artificial cracks with an identical width of $3 \mathrm{~mm}$. For reference convenience, the five defects are represented as $\mathrm{d} 1=3 \mathrm{~mm}, \mathrm{~d} 2=$ $5 \mathrm{~mm}, \mathrm{~d} 3=7 \mathrm{~mm}$, and $\mathrm{d} 4=9 \mathrm{~mm}$. Here, $\mathrm{d} 0=0 \mathrm{~mm}$ is tested for calibration and reference of the healthy state. The tag was directly placed on the surface of the sample without any gap. To study the influence of distance variation, the experiment was performed at three communication distances: $\mathrm{L} 1=20 \mathrm{~mm}$, L2 $=30 \mathrm{~mm}$, and $\mathrm{L} 3=40 \mathrm{~mm}$.

\section{B. Results and discussions}

Fig. 4 shows the tag's responses when it is directly placed on different cracks. It can be easily observed from Fig. 4(a) that the cracks have a significant influence on tag's performance, which can be wirelessly captured in the reader. The reader coil has a low quality factor; therefore, the overshoot due to the limit bandwidth is not apparent for the tag's response. The envelope 


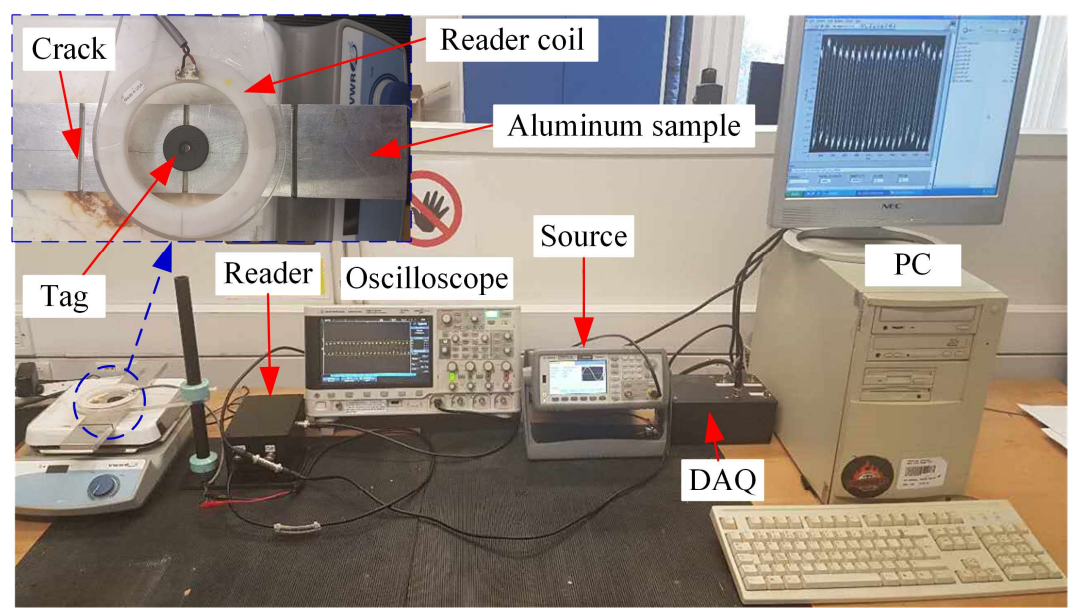

(a)

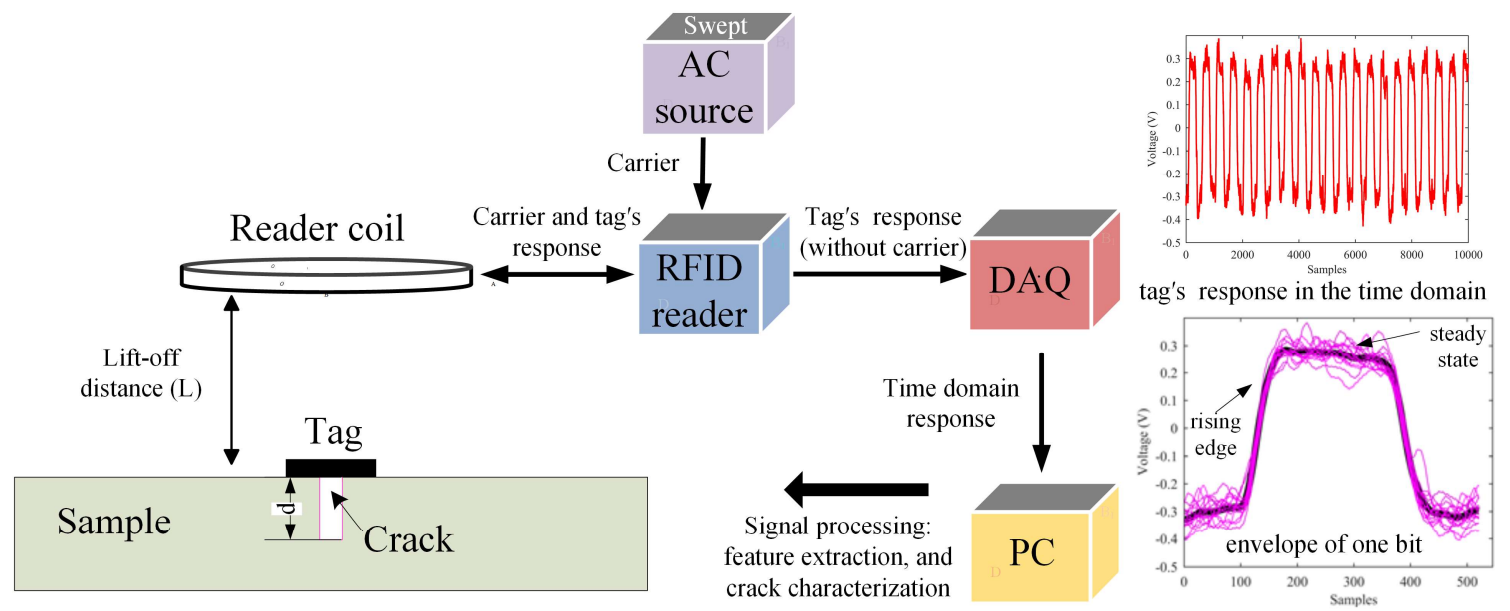

(b)

Fig. 3. Experimental setup for crack detection \& characterization based on LF RFID sensing system (a) setup and (b) block diagram.

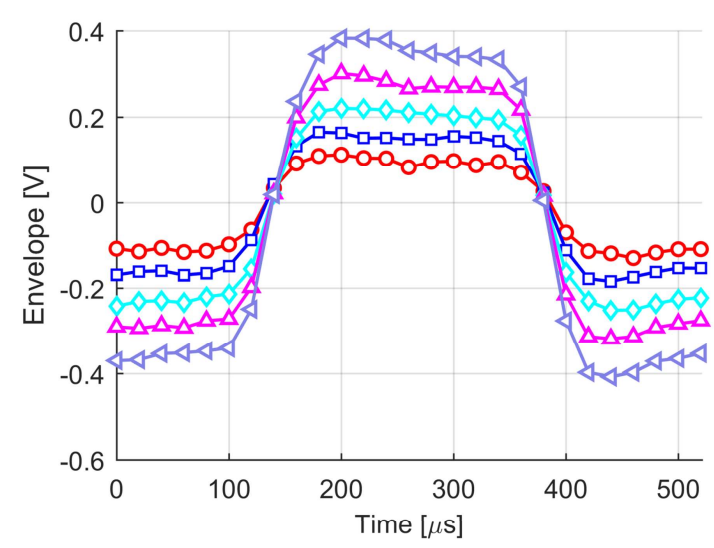

(a)

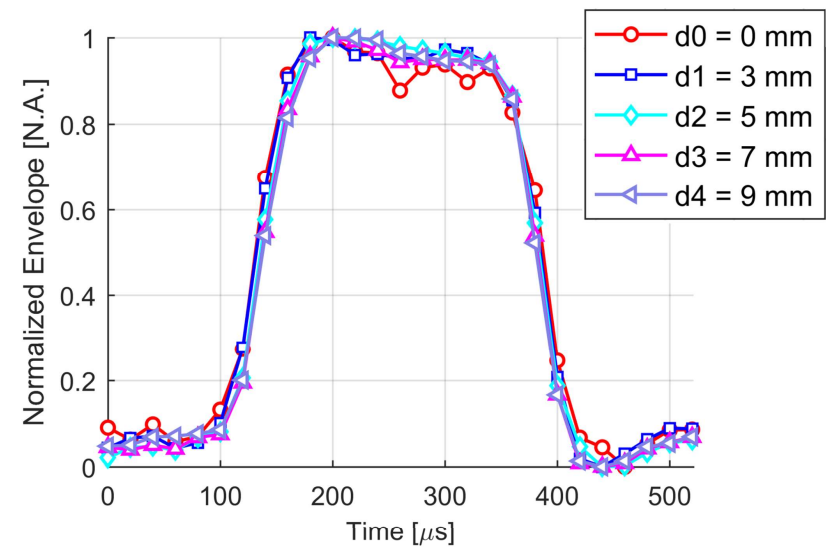

(b)

Fig. 4. Envelope of tag's response at $135 \mathrm{kHz}$ in a variation of crack depth when the communication distance is fixed to $\mathrm{L} 2=30 \mathrm{~mm}$ (a) before normalization and (b) after normalization.

needs to be normalized to suppress the influence of the mutual coupling, the result of which is shown in Fig. 4(b). However, it can be found that the feature of the rising edge is difficult to be used for crack monitoring after normalization.

The time-frequency feature representations of $\mathrm{P} 2 \mathrm{P}$ versus swept-frequency range are displayed in Figs. 5(a-c).
Comparing with the case in the air, we can find that a presence of metal leads to an approximate $14.0-\mathrm{kHz}$ increase of the resonant frequency. The increase of the communication distance has a negative influence in P2P but a negligible influence on the resonant frequency. The growth of crack, however, will constantly improve the coupling coefficient 


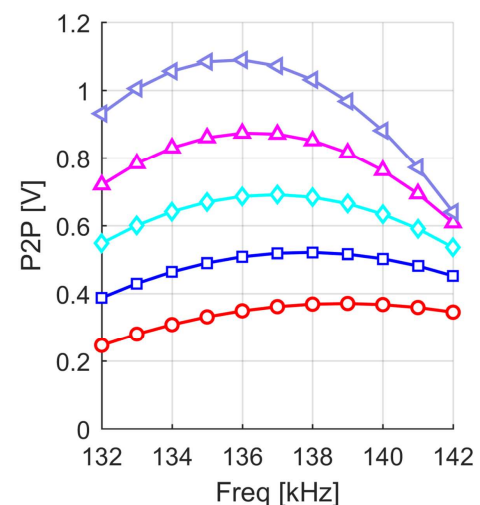

(a)

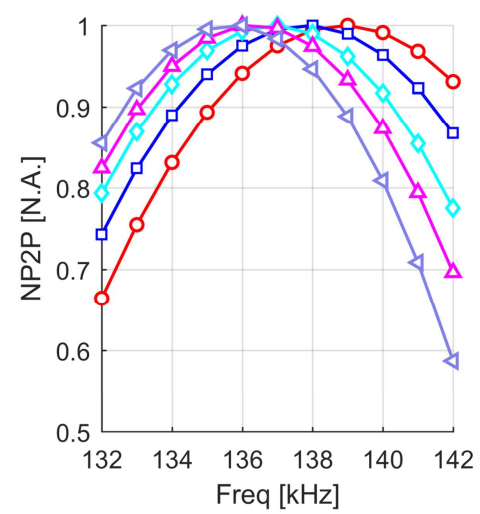

(d)

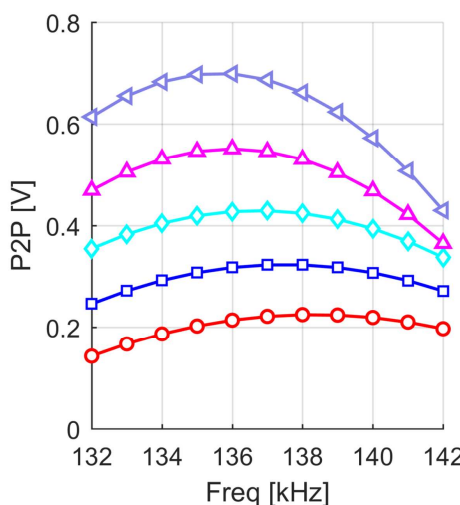

(b)

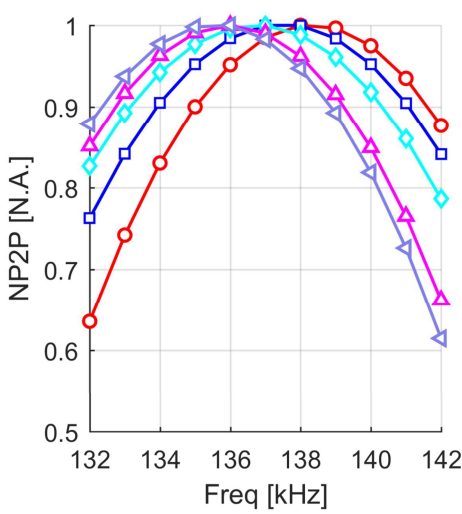

(e)

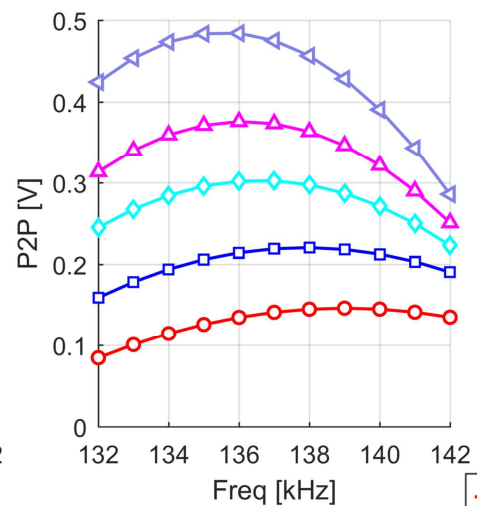

(c)

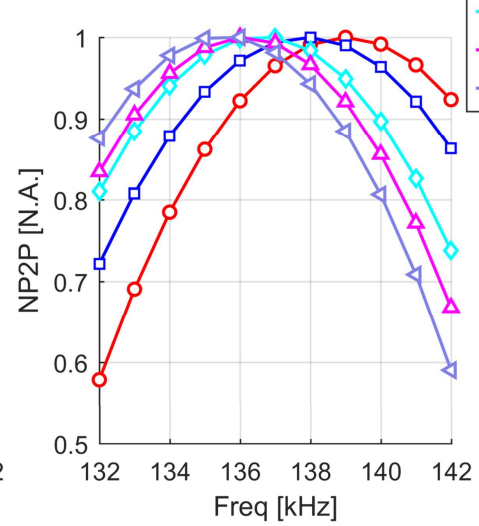

(f)

Fig. 5. Time-frequency feature representation of $\mathrm{P} 2 \mathrm{P}$ in the time-domain measurement over the swept-frequency range when the communication distances are L1 $=20 \mathrm{~mm}, \mathrm{~L} 2=30 \mathrm{~mm}$, and L3 $=40 \mathrm{~mm}$, respectively (a-c) before normalization and (d-f) after normalization.

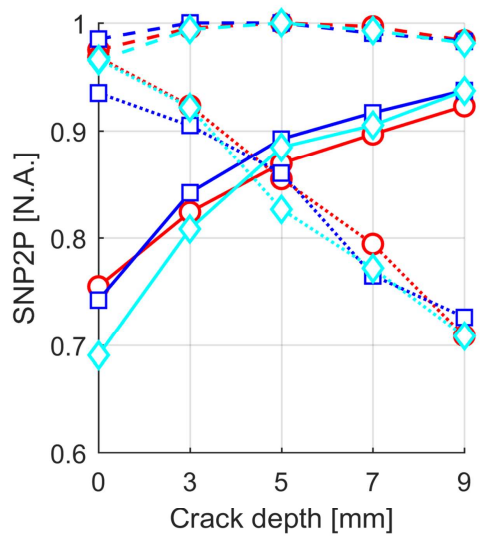

(a)

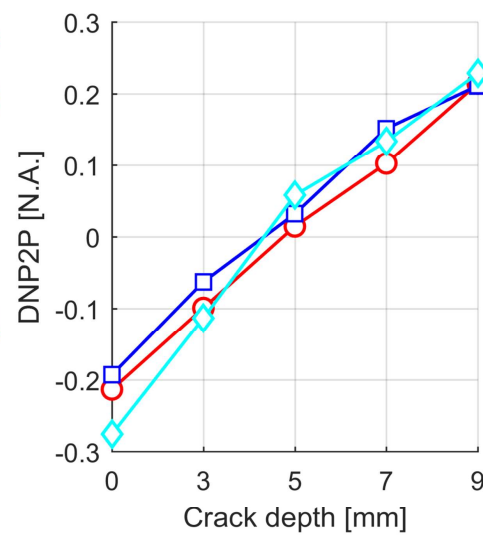

(b)

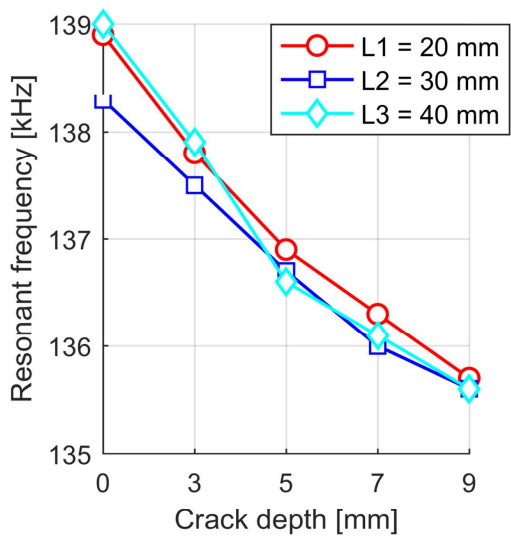

(c)

Fig. 6. Crack characterization at three communication distances: $\mathrm{L} 1=20 \mathrm{~mm}, \mathrm{~L} 2=30 \mathrm{~mm}$, and $\mathrm{L} 3=40 \mathrm{~mm}$ with and without feature fusion and selection (a) SNP2P1 (solid line), SNP2P2 (dash line), and SNP2P3 (dot line), (b) DNP2P, and (c) resonant frequency.

between the reader and tag coils, or the magnetic flux penetrating into the tag coil. As a result, an apparent increase in $\mathrm{P} 2 \mathrm{P}$ is observed. Meanwhile, the growth of crack will reduce the resonant frequency, which is contributed to the increase of self-inductance of the tag coil. It is worthy to mention that the curve shape of the time-frequency feature representation is determined by the quality factors of the reader and tag coils.

Through normalization to the maxima in the time-frequency feature representation, we can get the results of NP2P in the swept-frequency range, which are presented in Figs. 5(d-f). By comparing d0 in L3 and L1 at the frequency point of $132 \mathrm{kHz}$, we can find the variation of $\mathrm{P} 2 \mathrm{P}$ is from 0.08 to $0.25 \mathrm{~V}$ (3.1 times) before normalization. After normalization, the variation, however, is from 0.58 to 0.66 (1.1 times). Therefore, the influence of the communication distance can be essentially suppressed through normalization. Due to the resonance, we can find that the NP2P in the frequency lower than the resonant point has a positive coefficient with crack growth. Inversely, 
the NP2P in the frequency higher than the resonant point has a negative coefficient with crack growth.

Three types of features are extracted, fused, and selected for comparison. The first three features, represented as SNP2P1, SNP2P2, and SNP2P3, are NP2P at the single frequency point of $133.0 \mathrm{kHz}, 137.0 \mathrm{kHz}$, and $141.0 \mathrm{kHz}$, respectively. The fused feature of DNP2P is the second type, which is the differential of the first and third features, i.e., DNP2P = $\mathrm{SNP} 2 \mathrm{P} 1-\mathrm{SNP} 2 \mathrm{P} 3$. The third feature extracted in this paper is the resonant frequency.

Fig. 6(a) displays the results of first three features using single frequency. There exists a vague region near the transition point of negative and positive coefficients, in which the influence of crack cannot be discriminated by using NP2P, e.g., at a fixed frequency point of $137.0 \mathrm{kHz}$. Through feature fusion using DNP2P, the influence of the communication distance can be suppressed. This is verified in Fig. 6(b). As a result, the robustness of crack characterization is essentially enhanced. However, the resonant frequency at the presence of metal needs to be pre-determined for the feature selection. From Fig. 6(c), we can find that a variation of crack depth from $0 \mathrm{~mm}$ to $9 \mathrm{~mm}$ corresponds to a variation of resonant frequency from 135.5 $\mathrm{kHz}$ to $139.0 \mathrm{kHz}$, indicating a sensitivity of $0.39 \mathrm{kHz} / \mathrm{mm}$. An $\mathrm{mm}$ resolution can be achieved using the LF RFID sensing system in conjunction with the resonant frequency extracted from time-frequency feature representation.

The detection results in this work are in accordance to the PEC results of the same sample [44]. Because of a presence of tag between reader and defect, the RFID sensing system has a comparable sensitivity than the PEC technique even when the communication distance between the reader and tag is larger in an order of magnitude than the lift-off distance between the PEC probe and defect. It is worthy to mention that the change of communication distance is mathematically equivalent to the change of their orientation angles.

\section{CONCLUSIONS AND FUTURE WORK}

This paper studies the behavior of LF RFID sensing system at a presence of metal with defect. The influence of the mutual coupling between the reader and tag coils mixes with defects in the time-domain feature of $\mathrm{P} 2 \mathrm{P}$ and therefore affects robust defect detection and characterization. A sweep-frequency reader is developed for proof-of-concept study to coping with the mutual coupling variation. The resonant frequency is extracted from the time-frequency feature representation and used to separate influence of communication from sensing. The robustness of the LF RFID sensing system are enhanced.

It can be expected that the proximity of metal leads to a resonant frequency shift of the tag coil to the higher frequency region. This fact will reduce the quality factor of the tag coil as well as the mutual coupling between reader and tag coils, therefore, leading a reduction in the communication distance. This paper indicates that the crack growth will decrease the resonant frequency of the tag coil and improve the coupling between the reader and tag coils. That is to say, there is an increase in both self-inductance of the tag coil and mutual inductance between the reader and tag coils as crack grows.

For comprehensively evaluating the sensing performance of LF passive wireless RFID antenna sensors, a variety of cracks with different types and shapes will be investigated using the proposed method. The readout circuit with automatic resonant frequency tracking ability will also be developed to improve the response time and long-term repeatability and stability of the LF RFID sensing system.

\section{REFERENCES}

[1] M. D. Sangid, "The physics of fatigue crack initiation," Int. J. Fatigue, vol. 57, pp. 58-72, Dec. 2013.

[2] Y. Yao, S. T. E. Tung, and B. Glisic, "Crack detection and characterization techniques-An overview," Struct. Control Health Monit., vol. 21, no. 12, pp. 1387-1413, Dec. 2014.

[3] A. B. Noel, A. Abdaoui, T. Elfouly, M. H. Ahmed, A. Badawy, and M. S. Shehata, "Structural Health Monitoring Using Wireless Sensor Networks: A Comprehensive Survey," IEEE Commun. Surv. Tut., vol. 19, no. 3, pp. 1403-1423, thirdquarter 2017.

[4] J. Zhang, G. Y. Tian, A. M. J. Marindra, A. I. Sunny, and A. B. Zhao, "A Review of Passive RFID Tag Antenna-Based Sensors and Systems for Structural Health Monitoring Applications," Sensors-Basel, vol. 17, no. 2, Feb. 2017.

[5] A. Sophian, G. Y. Tian, and M. B. Fan, "Pulsed Eddy Current Non-destructive Testing and Evaluation: A Review," Chin. J. Mech. Eng. (Engl. Ed.), vol. 30, no. 3, pp. 500-514, May 2017.

[6] A. Sophian, G. Y. Tian, D. Taylor, and J. Rudlin, "A feature extraction technique based on principal component analysis for pulsed Eddy current NDT," NDT \& E Int., vol. 36, no. 1, pp. 37-41, Jan. 2003.

[7] I. Mohammad and H. Huang, "Monitoring fatigue crack growth and opening using antenna sensors," Smart Mater. Struct., vol. 19, no. 5, p. 055023 , May 2010.

[8] Z. Z. Meng and Z. Li, "RFID Tag as a Sensor - A Review on the Innovative Designs and Applications," Meas. Sci. Rev., vol. 16, no. 6, pp. 305-315, Dec. 2016.

[9] I. Mohammad and H. Y. Huang, "An Antenna Sensor for Crack Detection and Monitoring," Adv. Struct. Eng., vol. 14, no. 1, pp. 47-53, Feb. 2011.

[10] X. H. Yi, C. H. Cho, J. Cooper, Y. Wang, M. M. Tentzeris, and R. T. Leon, "Passive wireless antenna sensor for strain and crack sensing-electromagnetic modeling, simulation, and testing," Smart Mater. Struct., vol. 22, no. 8, p. 17, Aug. 2013.

[11] S. Caizzone and E. DiGiampaolo, "Wireless Passive RFID Crack Width Sensor for Structural Health Monitoring," IEEE Sensors J., vol. 15, no. 12, pp. 6767-6774, Dec. 2015.

[12] A. M. J. Marindra and G. Y. Tian, "Chipless RFID Sensor Tag for Metal Crack Detection and Characterization," IEEE Trans. Microw. Theory Tech., vol. 66, no. 5, pp. 2452-2462, May 2018.

[13] J. Zhang, G. Y. Tian, and A. B. Zhao, "Passive RFID sensor systems for crack detection \& characterization," NDT \& E Int., vol. 86, pp. 89-99, Mar. 2017.

[14] H. T. Cheng, S. Ebadi, and X. Gong, "A Low-Profile Wireless Passive Temperature Sensor Using Resonator/Antenna Integration Up to 1000 degrees C," IEEE Antennas Wireless Propag. Lett., vol. 11, pp. 369-372, 2012.

[15] D. Girbau, A. Ramos, A. Lazaro, S. Rima, and R. Villarino, "Passive Wireless Temperature Sensor Based on Time-Coded UWB Chipless RFID Tags," IEEE Trans. Microw. Theory Tech., vol. 60, no. 11, pp. 3623-3632, Nov. 2012.

[16] J. M. Boccard, T. Aftab, J. Hoppe, A. Yousaf, R. Hutter, and L. M. Reindl, "High-Resolution, Far-Field, and Passive Temperature Sensing up to 700 degrees C Using an Isolated ZST Microwave Dielectric Resonator," IEEE Sensors J., vol. 16, no. 3, pp. 715-722, Feb. 2016.

[17] J. Yao, F. M. Tchafa, A. Jain, S. Tjuatja, and H. Y. Huang, "Far-Field Interrogation of Microstrip Patch Antenna for Temperature Sensing Without Electronics," IEEE Sensors J., vol. 16, no. 19, pp. 7053-7060, Oct. 2016.

[18] J. Yao, J. Skilskyj, and H. Huang, "A compact, low-cost, real-time interrogation system for dynamic interrogation of microstrip patch antenna sensor," in Proc. SPIE 10598, Sensors and Smart Structures 
Technologies for Civil, Mechanical, and Aerospace Systems, Denver, CO, Mar. 2018.

[19] V. Chawla and D. S. Ha, "An overview of passive RFID," IEEE Commun. Mag., pp. 11-17, Sept. 2007.

[20] G. Y. Tian and A. Sophian, "Reduction of lift-off effects for pulsed eddy current NDT," NDT \& E Int., vol. 38, no. 4, pp. 319-324, Jun. 2005.

[21] T. Chen, G. Y. Tian, A. Sophian, and P. W. Que, "Feature extraction and selection for defect classification of pulsed eddy current NDT," NDT \& E Int., vol. 41, no. 6, pp. 467-476, Sept. 2008.

[22] G. Y. Tian, Y. Li, and C. Mandache, "Study of Lift-Off Invariance for Pulsed Eddy-Current Signals," IEEE Trans. Magn., vol. 45, no. 1, pp. 184-191, Jan. 2009.

[23] H. Zhang, R. Z. Yang, Y. Z. He, G. Y. Tian, L. X. Xu, and R. K. Wu, "Identification and characterisation of steel corrosion using passive high frequency RFID sensors," Measurement, vol. 92, pp. 421-427, Oct. 2016.

[24] J. Zhang and G. Y. Tian, "UHF RFID Tag Antenna-Based Sensing for Corrosion Detection \& Characterization Using Principal Component Analysis," IEEE Trans. Antennas Propag., vol. 64, no. 10, pp. 4405-4414, Oct. 2016.

[25] A. D. Metwally and S. F. Mahmoud, "Mutual coupling between loops on layered earth using images," IEEE Trans. Antennas Propag., vol. 32, no. 6, pp. 574-579, Jun. 1984.

[26] G. Y. Tian, J. Zhang, and Z. Z. Meng, "Passive Wireless RFID Sensors and Their Applications in Structural Health Monitoring," J. Nanjing Univ. Aeronaut. Astronaut., vol. 49, no. 4, pp. 453-460, Aug. 2017.

[27] A. A. Howling, P. Guittienne, R. Jacquier, and I. Furno, "Complex image method for RF antenna-plasma inductive coupling calculation in planar geometry. Part I: basic concepts," Plasma Sources Sci. Technol., vol. 24, no. 2015 , pp. $1-8$, Dec. 2015 .

[28] J. L. Schanen, C. Guerin, J. Roudet, and G. Meunier, "Influence of a conductive plane on loop inductance," IEEE Trans. Magn., vol. 31, no. 3, pp. 2127-2130, May 1995.

[29] M. R. Nabavi and S. N. Nihtianov, "Design Strategies for Eddy-Current Displacement Sensor Systems: Review and Recommendations," IEEE Sensors J., vol. 12, no. 12, pp. 3346-3355, Dec. 2012.

[30] C. Occhiuzzi and G. Marrocco, "Constrained-Design of Passive UHF RFID Sensor Antennas," IEEE Trans. Antennas Propag., vol. 61, no. 6, pp. 2972-2980, Jun. 2013.

[31] X. Zhou, R. Zhang, and C. K. Ho, "Wireless Information and Power Transfer: Architecture Design and Rate-Energy Tradeoff," IEEE Trans. Commun., vol. 61, no. 11, pp. 4754-4767, Nov. 2013

[32] Y. H. Sohn, B. H. Choi, E. S. Lee, G. C. Lim, G. H. Cho, and C. T. Rim, "General Unified Analyses of Two-Capacitor Inductive Power Transfer Systems: Equivalence of Current-Source SS and SP Compensations," IEEE Trans. Power Electron., vol. 30, no. 11, pp. 6030-6045, Nov. 2015.

[33] A. A. Eteng, S. K. A. Rahim, C. Y. Leow, S. Jayaprakasam, and B. W. Chew, "Low-power near-field magnetic wireless energy transfer links: A review of architectures and design approaches," Renew. Sust. Energ. Rev., vol. 77, pp. 486-505, Sept. 2017.

[34] C. Y. Xiao, K. Z. Wei, F. Liu, and Y. X. Ma, "Matching capacitance and transfer efficiency of four wireless power transfer systems via magnetic coupling resonance," Int. J. Circuit Theory Appl., vol. 45, no. 6, pp. 811-831, Jun. 2017.

[35] X. Z. Wei, Z. S. Wang, and H. F. Dai, "A Critical Review of Wireless Power Transfer via Strongly Coupled Magnetic Resonances," Energies, vol. 7, no. 7, pp. 4316-4341, Jul. 2014.

[36] A. P. Sample, D. A. Meyer, and J. R. Smith, "Analysis, Experimental Results, and Range Adaptation of Magnetically Coupled Resonators for Wireless Power Transfer," IEEE Trans. Ind. Electron., vol. 58, no. 2, pp. 544-554, Feb. 2011.

[37] K. Finkenzeller, RFID handbook: fundamentals and applications in contactless smart cards, radio frequency identification and near-field communication, 3rd ed.: John Wiley \& Sons, 2010.

[38] D. Ahn and S. Hong, "Wireless Power Transmission With Self-Regulated Output Voltage for Biomedical Implant," IEEE Trans. Ind. Electron., vol. 61, no. 5, pp. 2225-2235, May 2014.

[39] C. Carretero, "Coupling Power Losses in Inductive Power Transfer Systems With Litz-Wire Coils," IEEE Trans. Ind. Electron., vol. 64, no. 6, pp. 4474-4482, Jun. 2017.

[40] Q. A. Huang, L. Dong, and L. F. Wang, "LC Passive Wireless Sensors Toward a Wireless Sensing Platform: Status, Prospects, and Challenges," J. Microelectromech. Syst., vol. 25, no. 5, pp. 822-841, Oct. 2016.
[41] A. I. Sunny, G. Y. Tian, J. Zhang, and M. Pal, "Low frequency (LF) RFID sensors and selective transient feature extraction for corrosion characterisation," Sens. Actuator A-Phys., vol. 241, pp. 34-43, Apr. 2016.

[42] R. Nopper, R. Has, and L. Reindl, "A Wireless Sensor Readout System-Circuit Concept, Simulation, and Accuracy," IEEE Trans. Instrum. Meas., vol. 60, no. 8, pp. 2976-2983, Aug. 2011.

[43] Datasheet. (2017). HID ${ }^{\circledR}$ Volcano Tag ${ }^{\mathrm{TM}}$ RFID Tags: LF tag resistant to high temperatures. Available: https://www.hidglobal.com/products/rfid-tags/identification-technologi es/volcano-tag

[44] I. D. Adewale, "Multiple parameters based pulsed eddy current non-destructive testing and evaluation," PhD, School of Electrical and Electronic Engineering, Newcastle University, 2015. 